\title{
Systemic Inflammatory Markers Are Closely Associated with Atherogenic Lipoprotein Subfractions in Patients Undergoing Coronary Angiography
}

\author{
Yan Zhang, Sha Li, Rui-Xia Xu, Cheng-Gang Zhu, Yuan-Lin Guo, \\ Na-Qiong Wu, Jing Sun, and Jian-Jun Li

\begin{abstract}
Division of Dyslipidemia, State Key Laboratory of Cardiovascular Disease, Fu Wai Hospital, National Center for Cardiovascular
\end{abstract} \\ Diseases, Peking Union Medical College, Chinese Academy of Medical Sciences, BeiLiShi Road 167, Beijing 100037, China
}

Correspondence should be addressed to Jian-Jun Li; lijianjun938@126.com

Received 15 September 2015; Accepted 21 October 2015

Academic Editor: Consolato M. Sergi

Copyright (C) 2015 Yan Zhang et al. This is an open access article distributed under the Creative Commons Attribution License, which permits unrestricted use, distribution, and reproduction in any medium, provided the original work is properly cited.

\begin{abstract}
Objective. To investigate the relationship between inflammatory markers and atherogenic lipoprotein subfractions. Methods. We studied 520 eligible subjects who were not receiving any lipid-lowering therapy. The inflammatory markers including white blood cell (WBC) count, high-sensitivity C-reactive protein (hs-CRP), fibrinogen, erythrocyte sedimentation rate (ESR), and D-dimer were measured. A multimarker inflammatory index was developed. Low-density lipoprotein (LDL) and high-density lipoprotein (HDL) separation processes were performed using Lipoprint System. Results. In age- and sex-adjusted analysis, several inflammatory markers (WBC count, hs-CRP, fibrinogen, and ESR) were positively related to circulating non-HDL cholesterol and remnant cholesterol ( $p<0.05$, all). Among lipoprotein subfractions, we observed a positive association of inflammatory markers with very low-density lipoprotein cholesterol, small LDL cholesterol, and LDL score $(p<0.05$, all). Meanwhile, a negative association was detected between inflammatory markers and mean LDL particle size $(p<0.05)$ or large HDL cholesterol $(p<0.05)$. Moreover, we found that the relationships between multimarker index quartiles and small LDL cholesterol, LDL score, and mean LDL particle size were slightly stronger in patients with CAD. Conclusions. Systemic inflammatory markers are positively correlated with small LDL cholesterol and LDL score while being negatively linked with mean LDL particle size and large HDL cholesterol, highlighting the potential contribution to increased cardiovascular risk.
\end{abstract}

\section{Introduction}

Coronary artery disease (CAD) represents an important public health burden. Aside from the traditional risk factors, compelling evidence has now accumulated in support of inflammation as an important risk factor [1]. It has a pivotal role in all stages of atherosclerosis from endothelial dysfunction and plaque formation to plaque disruption and thrombosis [2]. Several inflammatory markers such as Creactive protein $(\mathrm{CRP})$ and fibrinogen have acquired prognostic significance $[3,4]$.

Although dyslipidemia has been recognized as the major cardiovascular risk factor and lowering of low-density lipoprotein (LDL) cholesterol has convincingly been shown to reduce cardiovascular events, there is still considerable remaining risk $[5,6]$. Indeed, LDL particles are a heterogeneous collection of particles which vary in potential pathologic properties such as size, density, and lipid composition [7]. Moreover, high-density lipoprotein (HDL) particles are much more heterogeneous in their size and composition than LDL. This may at least partly explain why attempts to reduce cardiovascular events by pharmacologically increasing HDL plasma levels have failed $[8,9]$. Hence, lipoprotein subfractions have emerged as a novel approach to assess the atherogenicity of lipoproteins.

There is a large body of evidence linking inflammatory status and dyslipidemia [10, 11]. Notably, it has been well established that inflammation is also able to affect lipoprotein metabolism [12]. Moreover, our previous data demonstrated positive associations between inflammatory markers and 
important cholesterol regulator, proprotein convertase subtilisin/kexin type 9 (PCSK9) $[13,14]$, which has also been found to be closely related to atherogenic lipoprotein subfractions $[15,16]$, However, whether there are certain relationships between inflammatory markers and lipoprotein subfractions has not been reported yet. To address this question, we studied five kinds of systemic inflammatory markers (white blood cell (WBC) count and its subsets, high-sensitivity CRP (hs-CRP), fibrinogen, erythrocyte sedimentation rate (ESR), and D-dimer) in a group of subjects scheduled for coronary angiography who were not receiving any lipidlowering therapy. Specifically, we hypothesized a presence of heterogeneity in the relationship of systemic inflammatory markers with atherogenic lipoprotein subfractions, which would aid our understanding of their interplay in the pathogenesis of atherosclerotic disease.

\section{Materials and Methods}

2.1. Study Design and Population. The study complied with the Declaration of Helsinki and was approved by the hospital's ethical review board (Fu Wai Hospital, National Center for Cardiovascular Diseases, Beijing, China). Each participant provided written, informed consent before enrollment.

In a group of subjects scheduled for coronary angiography because of angina-like chest pain and/or positive treadmill exercise test or clinically suspected CAD in our department, we selected 520 consecutive individuals who were not treated with lipid-lowering drugs (351 CAD and 169 non-CAD). Inclusion criteria were as follows: (1) having no treatment history of statins and/or other lipid-lowering drugs at least 3 months before entering the study; (2) having detailed clinical, laboratory data and well documented traditional cardiovascular risk factors; (3) having undergone coronary angiography. Exclusion criteria were subjects over 90 years, pregnancy or lactation, psychiatric disorder, the existence of any infectious or systematic inflammatory disease, acute coronary syndrome, serious heart failure or arrhythmia, significant hematologic disorders, thyroid dysfunction, severe liver dysfunction, and/or renal insufficiency and malignant tumors. The flowchart of the current study was shown in Figure 1.

Hypertension was defined as repeated blood pressure measurements $\geq 140 / 90 \mathrm{mmHg}$ (at least two times in different environments) or currently taking antihypertensive drugs. Diabetes mellitus (DM) was defined as a fasting serum glucose level $\geq 126 \mathrm{mg} / \mathrm{dL}$ in multiple determinations and/or the current use of medication for diabetes. Dyslipidemia was defined by medical history or the use of lipid-modulating medications in order to reduce lipids or fasting total cholesterol (TC) $\geq 200 \mathrm{mg} / \mathrm{dL}$ or triglyceride $(\mathrm{TG}) \geq 150 \mathrm{mg} / \mathrm{dL}$.

2.2. Biochemical and Clinical Analyses. Fasting blood samples were collected in precooled EDTA tubes at baseline from each patient. After centrifugation at $3000 \mathrm{rpm}$ for $15 \mathrm{~min}$ at $4^{\circ} \mathrm{C}$, all plasma aliquots were stored in our laboratory at $-80^{\circ} \mathrm{C}$ and were not thawed until use.
The concentrations of lipid profiles were determined by automatic biochemistry analyzer (Hitachi 7150, Tokyo, Japan). In detail, the LDL cholesterol concentration was analyzed by selective solubilization method (low-density lipid cholesterol test kit, Kyowa Medex, Tokyo). HDL cholesterol concentration was determined by a homogeneous method (Determiner L HDL, Kyowa Medex, Tokyo). Non-HDL cholesterol was calculated as TC minus HDL cholesterol. Remnant cholesterol was calculated as TC-HDL-C-LDL-C as previously reported [17]. The WBC count, neutrophil, lymphocyte, and monocyte differentials were determined using an automated blood cell counter (Beckman Coulter Ireland Inc., Mervue, Galway, Ireland). The plasma hs-CRP levels were determined using immunoturbidimetry (Beckman Assay 360, Bera, CA, USA). The fibrinogen levels were quantitatively measured by the method of Clauss and a Stago autoanalyzer with STA Fibrinogen kit (Diagnostica Stago, Taverny, France). The Westergren method was used for the measurement of ESR. Plasma D-dimer level was measured by Stago evolution (France).

2.3. LDL Subfraction Analysis. The cholesterol contents of LDL subfractions were determined electrophoretically by the use of high-resolution 3\% polyacrylamide gel tubes and the Lipoprint LDL System (Quantimetrix Corporation, Redondo Beach, CA, USA) according to the manufacturer's instructions as previously described [18]. Seven LDL subfractions were obtained. Subfraction 1 represented large LDL particles, subfraction 2 indicated intermediate LDL particles, and subfractions 3-7 were defined as small LDL particles. The cholesterol mass $(\mathrm{mg} / \mathrm{dL})$ of each lipoprotein subfraction, the mean LDL particle size $(\AA)$, and the proportion (\%) of the cholesterol mass of each lipoprotein subfraction over the TC mass were determined by this assay. The LDL score was calculated as the proportion of small LDL particles to the whole LDL area in our sample [19].

2.4. HDL Subfraction Analysis. Similar to LDL subfraction analysis, the cholesterol contents of HDL subfractions were also determined electrophoretically by the use of highresolution 3\% polyacrylamide gel tubes and the Lipoprint HDL System (Quantimetrix Corporation, Redondo Beach, CA, USA) as in our previously described work [20]. The relative area for each HDL subfraction was determined and multiplied by HDL cholesterol concentration of the sample to yield the amount of cholesterol for each band in $\mathrm{mg} / \mathrm{dL}$. Using this assay, HDL was divided into 10 subfractions. Subfractions 1-3 represented large HDL particles, subfractions 47 indicated intermediate HDL particles, and subfractions 810 meant small HDL particles.

2.5. Statistical Analysis. The data were expressed as the mean $\pm \mathrm{SD}$ for the continuous variables and the number (percentage) for the categorical variables. Student's $t$-test or Mann-Whitney $U$ test was used for the comparisons between continuous variables, and the chi-squared test was applied for the categorical variables between CAD and non-CAD group. Correlations between multiple inflammatory markers and 


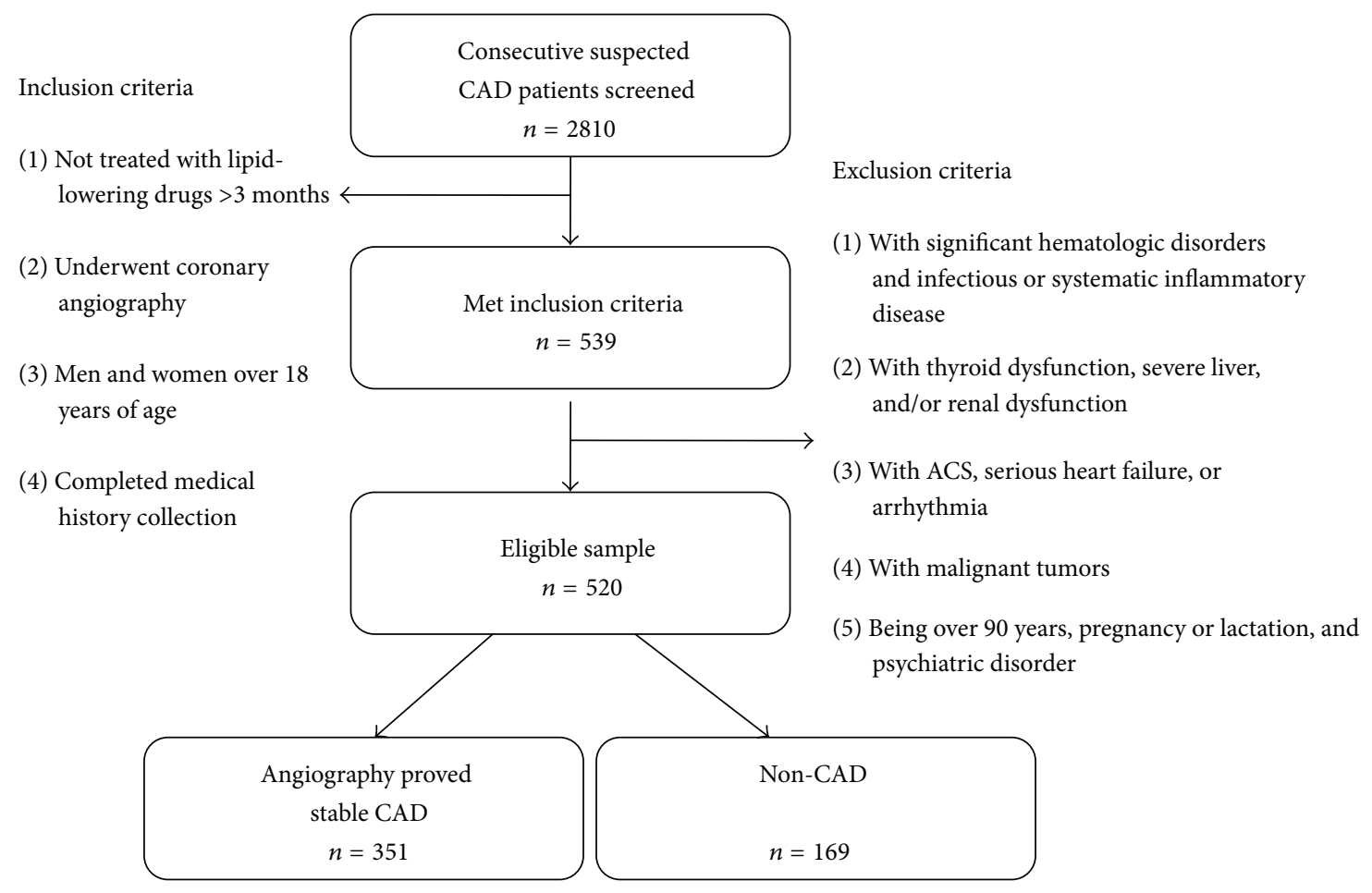

FIgURE 1: The flowchart of the current study.

lipoprotein subfractions were examined by partial correlation analysis with adjustments for age and sex. The chronic inflammatory activity can be assessed by a number of correlated parameters, such as WBC count, hs-CRP, fibrinogen, and ESR. Hence, we employed a principle component analysis to extract from the individual markers of inflammation (WBC count, hs-CRP, fibrinogen, and ESR) a single weighted multimarker inflammatory index. In the current study, only the first principle component was observed and no additional significant principal components were identified. Accordingly, we developed the overall multimarker inflammatory index by weighting the respective coefficients of each of the four inflammatory markers that contributed to the primary underlying factor (inflammation) as previously reported [21]. The general linear model was used for the comparison of lipoprotein subfractions according to multimarker index quartiles. The categorical variables were compared using the chi-squared test. A $p$ value of less than 0.05 was considered statistically significant. Statistical studies were carried out with the SPSS program (version 19.0, SPSS, Chicago, Illinois, USA).

\section{Results}

3.1. Baseline Characteristics. A total of 520 individuals were enrolled in the present study. The mean age of the study cohort was $56.6 \pm 10.0$ years and $335(64.4 \%)$ study participants were male. Among them, 351 (67.6\%) had significant angiographically documented CAD as having $>50 \%$ diameter stenosis in $\geq 1$ major epicardial coronary artery. The main demographic and clinical characteristics of the study subjects are listed in Table 1. As a result, we observed that the CAD group has relatively higher small LDL cholesterol levels $(9.0 \pm$ 9.8 versus $7.9 \pm 9.3 \mathrm{mg} / \mathrm{dL}, p=0.092)$ and $\mathrm{LDL}$ score $(0.14 \pm 0.13$ versus $0.12 \pm 0.13 \%, p=0.067)$ but smaller mean LDL particle size $(266.4 \pm 5.9$ versus $267.2 \pm 6.0 \AA$, $p=0.079$ ), although the difference does not reach statistical significance in the current analysis. Meanwhile, the CAD group has dramatically lower large HDL cholesterol (13.5 \pm 7.2 versus $15.1 \pm 7.7 \mathrm{mg} / \mathrm{dL}, p=0.027)$. In addition, several inflammatory markers are increased in patients with CAD, such as WBC count $\left(6.2 \pm 1.8\right.$ versus $5.9 \pm 1.4\left(\times 10^{9} / \mathrm{L}\right), p=$ $0.076)$, neutrophil count $\left(3.8 \pm 1.4\right.$ versus $3.5 \pm 1.1\left(\times 10^{9} / \mathrm{L}\right)$, $p=0.019)$, hs-CRP $(2.8 \pm 3.1$ versus $2.2 \pm 2.8 \mathrm{mg} / \mathrm{L}, p<0.001)$, and fibrinogen $(3.2 \pm 0.8$ versus $3.0 \pm 0.6 \mathrm{~g} / \mathrm{L}, p=0.001)$.

3.2. Correlations of Multiple Inflammatory Markers to Lipoprotein Subfractions. We next determined the strength of the relationship of multiple inflammatory markers with atherogenic lipoprotein subfractions. As shown in Table 2, after adjusting for age and sex, positive associations were observed between inflammatory markers and very lowdensity lipoprotein (VLDL) as well as intermediate-density lipoprotein (IDL). Among LDL subfractions, small LDL cholesterol was closely and positively related to WBC count ( $p<0.01)$, neutrophil count $(p<0.05)$, lymphocyte count $(p<0.01)$, hs-CRP $(p<0.01)$, fibrinogen $(p<0.001)$, and ESR $(p<0.05)$. Similar results were found between LDL score and inflammatory markers. However, the large LDL cholesterol, which has been supposed to be less atherogenic 
TABLE 1: Baseline characteristics.

\begin{tabular}{|c|c|c|c|c|}
\hline Characteristics & All subjects $(n=520)$ & $\mathrm{CAD}(n=351)$ & Non-CAD $(n=169)$ & $p$ value \\
\hline \multicolumn{5}{|l|}{ Coronary risk factors } \\
\hline Age (years) & $56.6 \pm 10.0$ & $57.9 \pm 9.7$ & $54.0 \pm 10.2$ & $<0.001$ \\
\hline Male, $\%(n)$ & $64.4(335)$ & $71.8(252)$ & $28.2(99)$ & $<0.001$ \\
\hline BMI $\left(\mathrm{kg} / \mathrm{m}^{2}\right)$ & $25.7 \pm 3.5$ & $25.9 \pm 3.4$ & $25.4 \pm 3.5$ & 0.147 \\
\hline Smoking, \% (n) & $43.5(226)$ & $50.1(176)$ & $29.6(50)$ & $<0.001$ \\
\hline Hypertension, \% (n) & $60.0(312)$ & $67.0(235)$ & $45.6(77)$ & $<0.001$ \\
\hline Diabetes mellitus, \% ( $n)$ & $21.7(113)$ & $25.1(88)$ & $14.8(25)$ & 0.009 \\
\hline Dyslipidemia, \% (n) & $67.7(352)$ & $70.7(248)$ & $61.5(104)$ & 0.045 \\
\hline Family history of CAD, \% $(n)$ & $16.0(83)$ & $17.9(63)$ & $11.8(20)$ & 0.096 \\
\hline \multicolumn{5}{|l|}{ Lipoprotein parameters } \\
\hline VLDL cholesterol (mg/dL) & $44.0 \pm 12.3$ & $44.0 \pm 10.8$ & $43.9 \pm 14.9$ & 0.253 \\
\hline IDL cholesterol (mg/dL) & $48.7 \pm 13.8$ & $48.3 \pm 13.4$ & $49.4 \pm 14.6$ & 0.606 \\
\hline LDL cholesterol (mg/dL) & $126.1 \pm 38.7$ & $125.7 \pm 38.4$ & $127.0 \pm 39.3$ & 0.723 \\
\hline Large LDL cholesterol & $28.7 \pm 10.1$ & $28.3 \pm 10.1$ & $29.4 \pm 10.1$ & 0.277 \\
\hline Intermediate LDL cholesterol & $20.5 \pm 9.5$ & $20.7 \pm 9.5$ & $20.1 \pm 9.4$ & 0.466 \\
\hline Small LDL cholesterol & $8.6 \pm 9.6$ & $9.0 \pm 9.8$ & $7.9 \pm 9.3$ & 0.092 \\
\hline LDL score $(\%)$ & $0.13 \pm 0.13$ & $0.14 \pm 0.13$ & $0.12 \pm 0.13$ & 0.067 \\
\hline Mean LDL particle size $(\AA)$ & $266.7 \pm 6.0$ & $266.4 \pm 5.9$ & $267.2 \pm 6.0$ & 0.079 \\
\hline HDL cholesterol (mg/dL) & $43.3 \pm 13.9$ & $42.5 \pm 14.2$ & $45.1 \pm 13.1$ & 0.054 \\
\hline Large HDL cholesterol & $14.0 \pm 7.4$ & $13.5 \pm 7.2$ & $15.1 \pm 7.7$ & 0.027 \\
\hline Intermediate HDL cholesterol & $20.9 \pm 6.5$ & $20.7 \pm 7.0$ & $21.2 \pm 5.3$ & 0.360 \\
\hline Small HDL cholesterol & $8.6 \pm 3.3$ & $8.5 \pm 3.4$ & $8.8 \pm 3.1$ & 0.318 \\
\hline \multicolumn{5}{|l|}{ Inflammatory markers } \\
\hline WBC count $\left(\times 10^{9} / \mathrm{L}\right)$ & $6.1 \pm 1.7$ & $6.2 \pm 1.8$ & $5.9 \pm 1.4$ & 0.076 \\
\hline Neutrophil count $\left(\times 10^{9} / \mathrm{L}\right)$ & $3.7 \pm 1.4$ & $3.8 \pm 1.4$ & $3.5 \pm 1.1$ & 0.019 \\
\hline Lymphocyte count $\left(\times 10^{9} / \mathrm{L}\right)$ & $1.9 \pm 0.6$ & $1.9 \pm 0.6$ & $2.0 \pm 0.5$ & 0.260 \\
\hline Monocyte count $\left(\times 10^{9} / \mathrm{L}\right)$ & $0.39 \pm 0.29$ & $0.40 \pm 0.34$ & $0.37 \pm 0.12$ & 0.147 \\
\hline hs-CRP (mg/L) & $2.6 \pm 9.8$ & $2.8 \pm 3.1$ & $2.2 \pm 2.8$ & $<0.001$ \\
\hline Fibrinogen $(\mathrm{g} / \mathrm{L})$ & $3.1 \pm 0.8$ & $3.2 \pm 0.8$ & $3.0 \pm 0.6$ & 0.001 \\
\hline $\operatorname{ESR}(\mathrm{mm} / \mathrm{h})$ & $9.8 \pm 9.7$ & $10.1 \pm 10.2$ & $9.1 \pm 8.6$ & 0.463 \\
\hline $\mathrm{D}$-dimer $(\mu \mathrm{g} / \mathrm{mL})$ & $0.37 \pm 0.44$ & $0.40 \pm 0.37$ & $0.33 \pm 0.56$ & 0.115 \\
\hline
\end{tabular}

Data are expressed as $\%(n)$, median (IQR), or mean \pm SD. BMI: body mass index; CAD: coronary artery disease; VLDL: very low-density lipoprotein; IDL: intermediate-density lipoprotein; LDL: low-density lipoprotein; HDL: high-density lipoprotein; WBC: white blood cell; hs-CRP: high sensitivity C-reactive protein; ESR: erythrocyte sedimentation rate.

than small LDL cholesterol, was not significantly linked with any inflammatory markers in the current study $(p>0.05$, all). We further assessed the correlation between inflammatory markers and mean LDL particle size. Interestingly, our data indicated a definitely negative association (WBC count: $p<$ 0.01; lymphocyte count: $p<0.01$; hs-CRP: $p<0.05$; fibrinogen: $p<0.01$; and ESR: $p<0.05$ ).

Additionally, in an analysis covering HDL subfractions, multiple inflammatory markers were correlated inversely with large HDL cholesterol (WBC count: $p<0.01$; lymphocyte count: $p<0.05$; hs-CRP: $p<0.05$; fibrinogen: $p<0.05$; and D-dimer: $p<0.05)$ but not with intermediate HDL cholesterol (only hs-CRP: $p<0.05$ ) and small HDL cholesterol $(p>0.05$, all).

3.3. Relation of Multimarker Inflammatory Index to Lipoprotein Subfractions. Of the individual inflammatory markers,
WBC count, hs-CRP, fibrinogen, and ESR were closely related to atherogenic lipoprotein subfractions; therefore, we extracted a multimarker inflammatory index weighting the coefficients of the four individual markers. Consequently, we divided this multimarker index into quartiles. As indicated in Table 3, in a model adjusting for age, sex, body mass index, hypertension, diabetes, smoking, and incidence of CAD, the levels of intermediate LDL cholesterol, small LDL cholesterol, and LDL score were dramatically increased while the mean LDL particle size was decreased according to multimarker index quartiles $(p<0.01$, all). Besides that, the large HDL cholesterol levels were markedly declined by multimarker index quartiles $(p<0.05)$.

3.4. Subgroup Analysis in Patients with or without CAD. Given that larger percentage of patients with CAD tended to have more severe inflammation (Figure 2), we further 
TABLE 2: Age- and sex-adjusted correlations between lipoprotein subfractions and inflammatory markers.

\begin{tabular}{lcccccccc}
\hline & $\begin{array}{c}\text { WBC } \\
\text { count }\end{array}$ & $\begin{array}{c}\text { Neutrophil } \\
\text { count }\end{array}$ & $\begin{array}{c}\text { Lymphocyte } \\
\text { count }\end{array}$ & $\begin{array}{c}\text { Monocyte } \\
\text { count }\end{array}$ & hs-CRP & Fibrinogen & ESR & D-dimer \\
\hline VLDL cholesterol (mg/dL) & $0.133^{* *}$ & $0.112^{*}$ & $0.124^{* *}$ & -0.019 & $0.129^{* *}$ & $0.189^{* * *}$ & $0.154^{* * *}$ & -0.055 \\
IDL cholesterol (mg/dL) & 0.055 & 0.080 & 0.009 & -0.053 & $0.115^{* *}$ & $0.129^{* *}$ & $0.090^{*}$ & -0.042 \\
LDL cholesterol (mg/dL) & 0.078 & 0.079 & 0.053 & -0.028 & $0.112^{*}$ & $0.090^{*}$ & 0.020 & -0.056 \\
$\quad$ Large LDL cholesterol & -0.054 & -0.020 & -0.075 & -0.059 & 0.008 & -0.031 & -0.064 & -0.008 \\
$\quad$ Intermediate LDL cholesterol & $0.094^{*}$ & 0.072 & $0.102^{*}$ & 0.001 & $0.153^{* * *}$ & $0.095^{*}$ & 0.045 & -0.077 \\
Small LDL cholesterol & $0.128^{* *}$ & $0.099^{*}$ & $0.120^{* *}$ & 0.007 & $0.121^{* *}$ & $0.141^{* * *}$ & $0.111^{*}$ & -0.054 \\
LDL score (\%) & $0.124^{* *}$ & $0.095^{*}$ & $0.111^{*}$ & 0.009 & $0.099^{*}$ & $0.139^{* *}$ & $0.125^{* *}$ & -0.057 \\
Mean LDL particle size $(\AA)$ & $-0.115^{* *}$ & -0.084 & $-0.117^{* *}$ & -0.016 & $-0.096^{*}$ & $-0.117^{* *}$ & $-0.111^{*}$ & 0.071 \\
HDL cholesterol (mg/dL) & $-0.093^{*}$ & -0.064 & -0.072 & -0.059 & $-0.106^{*}$ & -0.075 & -0.034 & 0.063 \\
$\quad$ Large HDL cholesterol & $-0.123^{* *}$ & $-0.089^{*}$ & $-0.104^{*}$ & -0.052 & -0.085 & $-0.092^{*}$ & -0.060 & $0.104^{*}$ \\
$\quad$ Intermediate HDL cholesterol & -0.077 & -0.055 & -0.053 & -0.053 & $-0.090^{*}$ & -0.047 & -0.029 & 0.042 \\
Small HDL cholesterol & -0.013 & -0.006 & -0.001 & -0.036 & -0.085 & -0.057 & 0.004 & -0.061 \\
\hline
\end{tabular}

Partial correlations are shown. All the correlations were adjusted for age and sex. VLDL: very low-density lipoprotein; IDL: intermediate-density lipoprotein; LDL: low-density lipoprotein; HDL: high-density lipoprotein; WBC: white blood cell; hs-CRP: high sensitivity C-reactive protein; ESR: erythrocyte sedimentation rate.

${ }^{*} p<0.05$.

${ }^{* *} p<0.01$.

*** $p<0.001$

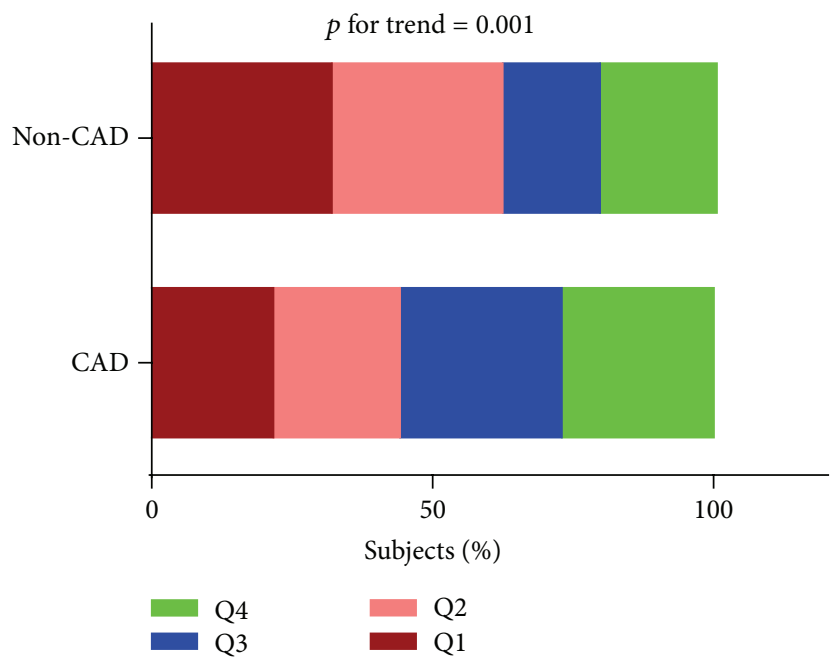

FIGURE 2: The distribution of subjects in CAD and non-CAD group across multimarker inflammatory index quartiles. Chi-squared test was performed.

performed the subgroup analysis to explore the association between inflammation and atherogenic lipoprotein subfractions in patients with or without CAD. As indicated in Figure 3, we found positive associations of multimarker index quartiles with small LDL cholesterol (CAD: $\beta=0.183, p=$ 0.001; non-CAD: $\beta=0.159, p=0.039$ ) and LDL score (CAD: $\beta=0.176, p=0.001$; non-CAD: $\beta=0.169, p=$ 0.029 ) and negative associations with mean LDL particle size (CAD: $\beta=-0.163, p=0.002$; non-CAD: $\beta=-0.160$, $p=0.039)$. Although the relationships were both significant in patients with or without CAD, the former tended to be slightly stronger in the present study.

\section{Discussion}

The present study confirms the low-grade systemic inflammatory markers are related to features of the circulating cholesterol levels. More importantly, the main and novel findings are that (1) multiple systemic inflammatory markers are positively correlated with the most atherogenic lipoprotein subfractions, such as small LDL cholesterol and LDL score; (2) they are negatively linked with mean LDL particle size; (3) and they are inversely related to the antiatherogenic subfraction, large HDL cholesterol. These findings suggest that the mutual interplay may be a potential major contributor in the development of atherosclerotic disease.

Although the notion that elevated inflammatory markers increase the risk of cardiovascular disease (CVD) has been increasingly recognized [22, 23], underlying mechanisms and pathways remain to be elucidated. Specifically, inflammation and dyslipidemia are well established cardiovascular risk factors and closely associated with each other. However, it remains unclear with regard to which comes first in the atherosclerotic process. As reported, inflammation could affect lipoprotein metabolism [12], which is reflected by decreased plasma HDL-C levels and impaired atheroprotective HDL functions [24]. Additionally, it also has been suggested that the relationship between inflammatory markers and atherosclerosis is independent of plasma lipoprotein levels [25]. Conversely, the presence of dyslipidemia itself may in turn further stimulate the inflammatory process [26]. There are evidences that inflammation could be elicited by modified lipoproteins such as oxidized LDL [27], as well as 
TABLE 3: Relationship of LDL and HDL subfractions with multimarker inflammatory index.

\begin{tabular}{|c|c|c|c|c|c|}
\hline \multicolumn{6}{|c|}{ Multimarker inflammatory index } \\
\hline 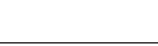 & $\mathrm{Q} 1(n=130)$ & $\mathrm{Q} 2(n=130)$ & Q3 $(n=130)$ & $\mathrm{Q} 4(n=130)$ & $p$ for trend \\
\hline \multicolumn{6}{|c|}{ LDL subfractions } \\
\hline \multicolumn{6}{|c|}{ Large LDL cholesterol (mg/dL) } \\
\hline Model 1 & $30.07 \pm 0.89$ & $27.92 \pm 0.89$ & $28.44 \pm 0.89$ & $28.03 \pm 0.89$ & 0.289 \\
\hline Model 2 & $30.36 \pm 0.89$ & $27.84 \pm 0.89$ & $28.30 \pm 0.89$ & $27.96 \pm 0.89$ & 0.157 \\
\hline Model 3 & $30.01 \pm 0.91$ & $27.89 \pm 0.89$ & $28.53 \pm 0.91$ & $28.06 \pm 0.90$ & 0.338 \\
\hline \multicolumn{6}{|c|}{ Intermediate LDL cholesterol (mg/dL) } \\
\hline Model 1 & $18.02 \pm 0.83$ & $20.76 \pm 0.83$ & $21.19 \pm 0.83$ & $22.02 \pm 0.83$ & 0.005 \\
\hline Model 2 & $18.03 \pm 0.84$ & $20.62 \pm 0.83$ & $21.29 \pm 0.84$ & $22.06 \pm 0.83$ & 0.005 \\
\hline Model 3 & $18.06 \pm 0.85$ & $20.72 \pm 0.83$ & $21.23 \pm 0.85$ & $22.01 \pm 0.83$ & 0.009 \\
\hline \multicolumn{6}{|c|}{ Small LDL cholesterol (mg/dL) } \\
\hline Model 1 & $5.87 \pm 0.87$ & $8.92 \pm 0.87$ & $9.46 \pm 0.87$ & $10.89 \pm 0.87$ & 0.001 \\
\hline Model 2 & $5.73 \pm 0.87$ & $8.72 \pm 0.87$ & $9.69 \pm 0.87$ & $11.00 \pm 0.87$ & $<0.001$ \\
\hline Model 3 & $5.79 \pm 0.90$ & $8.74 \pm 0.87$ & $9.64 \pm 0.89$ & $10.98 \pm 0.87$ & 0.001 \\
\hline \multicolumn{6}{|c|}{ LDL score (\%) } \\
\hline Model 1 & $0.09 \pm 0.01$ & $0.14 \pm 0.01$ & $0.15 \pm 0.01$ & $0.16 \pm 0.01$ & $<0.001$ \\
\hline Model 2 & $0.09 \pm 0.01$ & $0.13 \pm 0.01$ & $0.15 \pm 0.01$ & $0.16 \pm 0.01$ & $<0.001$ \\
\hline Model 3 & $0.10 \pm 0.01$ & $0.13 \pm 0.01$ & $0.15 \pm 0.01$ & $0.16 \pm 0.01$ & 0.001 \\
\hline \multicolumn{6}{|c|}{ Mean LDL particle size $(\AA)$} \\
\hline Model 1 & $268.42 \pm 0.52$ & $266.38 \pm 0.52$ & $266.21 \pm 0.52$ & $265.51 \pm 0.52$ & 0.001 \\
\hline Model 2 & $268.54 \pm 0.53$ & $266.44 \pm 0.52$ & $266.09 \pm 0.53$ & $265.45 \pm 0.52$ & $<0.001$ \\
\hline Model 3 & $268.39 \pm 0.54$ & $266.45 \pm 0.52$ & $266.18 \pm 0.53$ & $265.49 \pm 0.52$ & 0.001 \\
\hline \multicolumn{6}{|c|}{ HDL subfractions } \\
\hline \multicolumn{6}{|c|}{ Large HDL cholesterol (mg/dL) } \\
\hline Model 1 & $15.29 \pm 0.64$ & $13.57 \pm 0.64$ & $14.03 \pm 0.64$ & $13.02 \pm 0.64$ & 0.080 \\
\hline Model 2 & $15.97 \pm 0.61$ & $13.75 \pm 0.61$ & $13.44 \pm 0.61$ & $12.73 \pm 0.61$ & 0.002 \\
\hline Model 3 & $15.33 \pm 0.60$ & $13.62 \pm 0.59$ & $13.95 \pm 0.60$ & $13.04 \pm 0.59$ & 0.049 \\
\hline \multicolumn{6}{|c|}{ Intermediate HDL cholesterol (mg/dL) } \\
\hline Model 1 & $21.69 \pm 0.57$ & $20.70 \pm 0.57$ & $20.27 \pm 0.57$ & $20.65 \pm 0.57$ & 0.329 \\
\hline Model 2 & $22.07 \pm 0.56$ & $20.71 \pm 0.56$ & $20.02 \pm 0.56$ & $20.52 \pm 0.56$ & 0.066 \\
\hline Model 3 & $21.96 \pm 0.57$ & $20.68 \pm 0.56$ & $20.08 \pm 0.57$ & $20.58 \pm 0.56$ & 0.129 \\
\hline \multicolumn{6}{|c|}{ Small HDL cholesterol (mg/dL) } \\
\hline Model 1 & $8.52 \pm 0.29$ & $8.84 \pm 0.29$ & $8.49 \pm 0.29$ & $8.40 \pm 0.30$ & 0.740 \\
\hline Model 2 & $8.56 \pm 0.30$ & $8.79 \pm 0.29$ & $8.50 \pm 0.30$ & $8.40 \pm 0.29$ & 0.815 \\
\hline Model 3 & $8.63 \pm 0.30$ & $8.80 \pm 0.29$ & $8.42 \pm 0.30$ & $8.37 \pm 0.29$ & 0.729 \\
\hline
\end{tabular}

Values were obtained from general linear models. Model 1 was unadjusted. Model 2 was adjusted for age and sex. Model 3 was additionally adjusted for BMI, hypertension, diabetes, smoking, and incidence of CAD. BMI: body mass index; CAD: coronary artery disease; LDL: low-density lipoprotein; HDL: highdensity lipoprotein.

the TG-rich lipoprotein remnants [28]. Thus, the interplay between inflammation and lipid metabolism at multiple levels may exacerbate the development of atherosclerosis, resulting in a vicious cycle. However, the traditionally measured cholesterol levels in LDL and HDL particles could not capture all the high LDL or low HDL related risks $[29,30]$ and other alternative measures reflecting the particle have emerged in multiple studies.

Recently, lipoprotein subfractions have been suggested as a new cardiovascular risk strategy [31]. Experimental and turnover studies have raised the possibility that small LDL may be more atherogenic than buoyant LDL [7]. The Atherosclerosis Risk in Communities study including 11419 participants revealed that small dense LDL cholesterol was associated with the incident CAD [32]. Moreover, Nishikura et al. conducted a study including 190 consecutive CAD patients. During a seven-year follow-up period, small dense LDL cholesterol has been supposed to be a very promising biomarker in predicting future cardiovascular events [33]. Despite the cholesterol levels in LDL subfractions, mean LDL particle size has also been indicated to be closely related to cardiovascular mortality [34]. Currently, the link of HDL subclasses to prognosis remains controversial. Most studies including our data tended to support the idea that decreased large HDL-C level may be more atherogenic than other HDL subfractions $[35,36]$. 


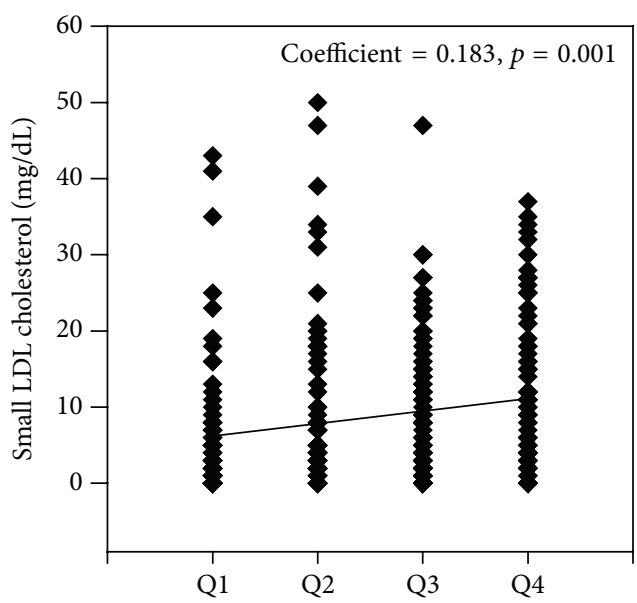

(a) $\mathrm{CAD}$

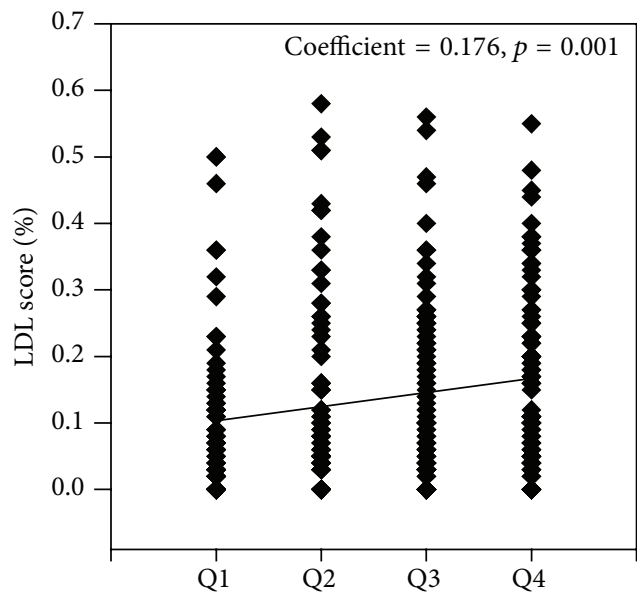

(c) $\mathrm{CAD}$

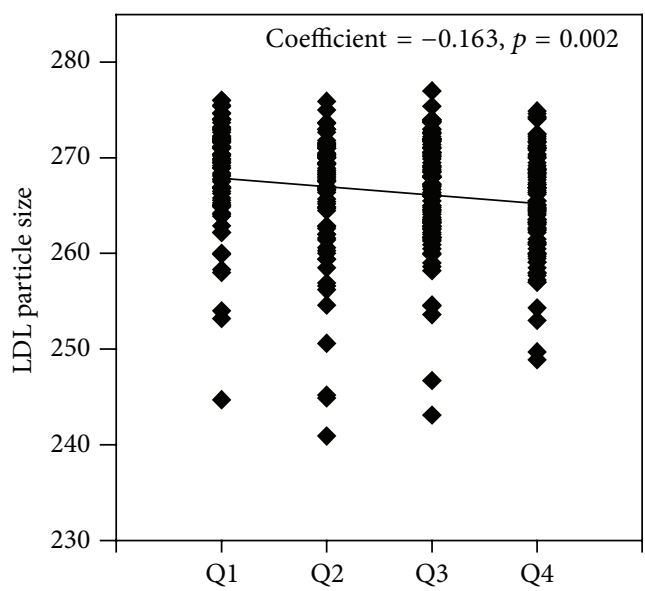

(e) $\mathrm{CAD}$

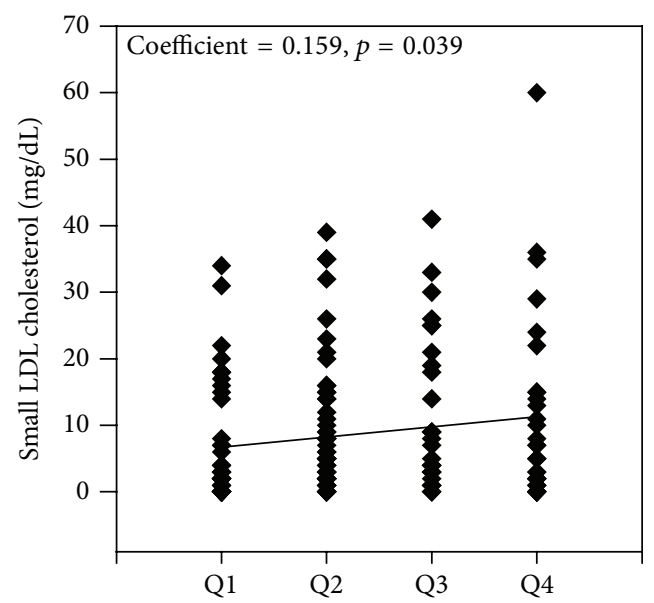

(b) Non-CAD

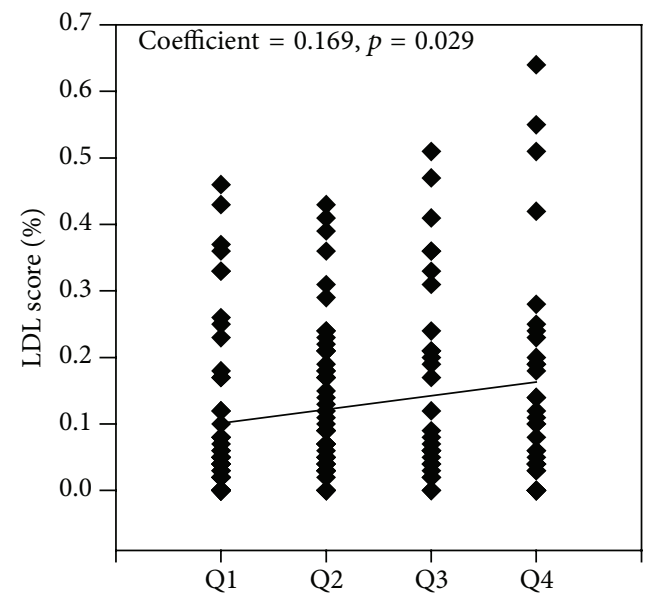

(d) Non-CAD

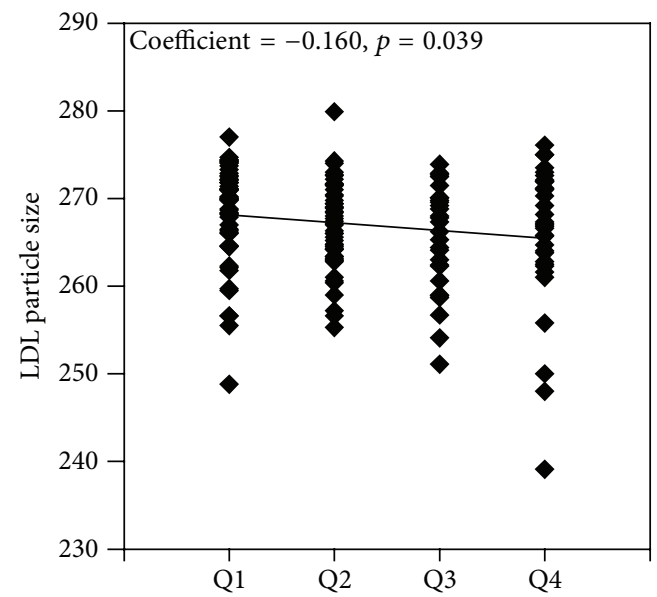

(f) Non-CAD

FIGURE 3: Relation of small LDL-C, LDL score, and mean LDL particle size to the quartiles of multimarker inflammatory index in patients with or without CAD ( $(a, b)$ small LDL-C; $(c, d)$ LDL score; $(e, f)$ mean LDL particle size). Simple linear regression analysis was applied.

In view of these heterogeneous and complex relationships, it is important to characterize the role of individual markers of inflammation in relation to different lipoprotein subfractions. In the present study, we employed five kinds of inflammatory markers reflecting the chronic systemic inflammatory activity and found that most of the inflammatory markers are positively associated with atherogenic lipoprotein subfractions in patients that underwent coronary 
angiography. Of additional importance, we derived a composite marker of inflammation from a principle component analysis. In doing so, we were able to combine each of the individual markers into a single component, thereby reflecting a general marker of inflammation, permitting us to retain most of the information attributed to each marker. As a result, we observed that the atherogenic lipoprotein subfractions were significantly increased by multimarker index in patients with or without CAD, although it was slightly stronger in the CAD group. These novel findings may suggest that the correlation between inflammatory markers and atherogenic lipoprotein subfractions was stable and persistent and mildly modified by CAD status. Nonetheless, future investigations will have to verify these relationships in different populations and address the exact mechanisms.

The present study is not without limitations. First, it included patients scheduled for angiography. This hospitalbased population may not be representative of a random population sample. Second, it was a cross-sectional study, so it was difficult to identify the causal or temporal relationship. Finally, we estimated the lipoprotein subfractions with Lipoprint System and the use of other methods should be investigated in the future.

\section{Conclusion}

In summary, systemic inflammatory markers are positively correlated with small LDL cholesterol and LDL score while being negatively linked with mean LDL particle size and large HDL cholesterol, highlighting the potential contribution to increased cardiovascular risk.

\section{Conflict of Interests}

The authors have no conflict of interests to disclose.

\section{Acknowledgments}

This work was partially supported by the National Natural Science Foundation of China (81070171, 81241121), the Specialized Research Fund for the Doctoral Program of Higher Education of China (20111106110013), the Capital Special Foundation of Clinical Application Research (Z121107001012015), the Capital Health Development Fund (2011400302), and the Beijing Natural Science Foundation (7131014) awarded to Dr. Jian-Jun Li, M.D. and Ph.D. holder.

\section{References}

[1] G. Biasillo, M. Leo, R. Della Bona, and L. M. Biasucci, "Inflammatory biomarkers and coronary heart disease: from bench to bedside and back," Internal and Emergency Medicine, vol. 5, no. 3, pp. 225-233, 2010.

[2] G. K. Hansson, "Inflammation, atherosclerosis, and coronary artery disease," The New England Journal of Medicine, vol. 352, no. 16, pp. 1626-1695, 2005.

[3] C. M. Ballantyne, R. C. Hoogeveen, H. Bang et al., "Lipoproteinassociated phospholipase $\mathrm{A}_{2}$, high-sensitivity C-reactive protein, and risk for incident coronary heart disease in middle-aged men and women in the Atherosclerosis Risk in Communities (ARIC) study," Circulation, vol. 109, no. 7, pp. 837-842, 2004.

[4] D. J. Eapen, P. Manocha, R. S. Patel et al., "Aggregate risk score based on markers of inflammation, cell stress, and coagulation is an independent predictor of adverse cardiovascular outcomes," Journal of the American College of Cardiology, vol. 62, no. 4, pp. 329-337, 2013.

[5] Heart Protection Study Collaborative Group, "MRC/BHF Heart Protection Study of cholesterol lowering with simvastatin in 20536 high-risk individuals: a randomised placebocontrolled trial," The Lancet, vol. 360, no. 9326, pp. 7-22, 2002.

[6] C. Baigent, L. Blackwell, J. Emberson et al., "Efficacy and safety of more intensive lowering of LDL cholesterol: a meta-analysis of data from 170,000 participants in 26 randomised trials," The Lancet, vol. 376, pp. 1670-1681, 2010.

[7] M. R. Diffenderfer and E. J. Schaefer, "The composition and metabolism of large and small LDL," Current Opinion in Lipidology, vol. 25, no. 3, pp. 221-226, 2014.

[8] B. Simic, M. Hermann, S. G. Shaw et al., "Torcetrapib impairs endothelial function in hypertension," European Heart Journal, vol. 33, no. 13, pp. 1615-1624, 2012.

[9] G. G. Schwartz, A. G. Olsson, M. Abt et al., "Effects of dalcetrapib in patients with a recent acute coronary syndrome," The New England Journal of Medicine, vol. 367, no. 22, pp. 20892099, 2012.

[10] D. Tousoulis, C. Antoniades, and C. Stefanadis, "Assessing inflammatory status in cardiovascular disease," Heart, vol. 93, no. 8, pp. 1001-1007, 2007.

[11] M. Navab, N. Gharavi, and A. D. Watson, "Inflammation and metabolic disorders," Current Opinion in Clinical Nutrition and Metabolic Care, vol. 11, no. 4, pp. 459-464, 2008.

[12] W. Khovidhunkit, M.-S. Kim, R. A. Memon et al., "Effects of infection and inflammation on lipid and lipoprotein metabolism: mechanisms and consequences to the host," Journal of Lipid Research, vol. 45, no. 7, pp. 1169-1196, 2004.

[13] S. Li, Y.-L. Guo, R.-X. Xu et al., "Association of plasma PCSK9 levels with white blood cell count and its subsets in patients with stable coronary artery disease," Atherosclerosis, vol. 234, no. 2, pp. 441-445, 2014.

[14] Y. Zhang, C.-G. Zhu, R.-X. Xu et al., "Relation of circulating PCSK9 concentration to fibrinogen in patients with stable coronary artery disease," Journal of Clinical Lipidology, vol. 8, no. 5, pp. 494-500, 2014.

[15] R.-X. Xu, S. Li, Y. Zhang et al., "Relation of plasma PCSK9 levels to lipoprotein subfractions in patients with stable coronary artery disease," Lipids in Health and Disease, vol. 13, article 188, 2014.

[16] M. Guardiola, N. Plana, D. Ibarretxe et al., "Circulating PCSK9 levels are positively correlated with NMR-assessed atherogenic dyslipidaemia in patients with high cardiovascular risk," Clinical Science, vol. 128, no. 12, pp. 877-882, 2015.

[17] R. McPherson, "Remnant cholesterol: 'Non-(HDL-C + LDL-C)' as a coronary artery disease risk factor," Journal of the American College of Cardiology, vol. 61, no. 4, pp. 437-439, 2013.

[18] D. M. Hoefner, S. D. Hodel, J. F. O’Brien et al., “Development of a rapid, quantitative method for LDL subfractionation with use of the Quantimetrix Lipoprint LDL System," Clinical Chemistry, vol. 47, no. 2, pp. 266-274, 2001.

[19] Y. Zhang, R.-X. Xu, S. Li et al., "Lipoprotein subfractions partly mediate the association between serum uric acid and coronary artery disease," Clinica Chimica Acta, vol. 441, pp. 109-114, 2015. 
[20] R.-X. Xu, Y.-L. Guo, X.-L. Li, S. Li, and J.-J. Li, "Impact of short-term low-dose atorvastatin on low-density lipoprotein and high-density lipoprotein subfraction phenotype," Clinical and Experimental Pharmacology and Physiology, vol. 41, no. 7, pp. 475-481, 2014.

[21] B. Ó Hartaigh, J. A. Bosch, D. Carroll et al., "Evidence of a synergistic association between heart rate, inflammation, and cardiovascular mortality in patients undergoing coronary angiography," European Heart Journal, vol. 34, no. 12, pp. 932941, 2013.

[22] R. P. Tracy, R. N. Lemaitre, B. M. Psaty et al., "Relationship of C-reactive protein to risk of cardiovascular disease in the elderly. Results from the Cardiovascular Health Study and the Rural Health Promotion Project," Arteriosclerosis, Thrombosis, and Vascular Biology, vol. 17, no. 6, pp. 1121-1127, 1997.

[23] Y. Zhang, C.-G. Zhu, Y.-L. Guo et al., "Higher fibrinogen level is independently linked with the presence and severity of newonset coronary atherosclerosis among han Chinese population," PLoS ONE, vol. 9, no. 11, Article ID el13460, 2014.

[24] F. C. McGillicuddy, M. L. La Moya, C. C. Hinkle et al., "Inflammation impairs reverse cholesterol transport in vivo," Circulation, vol. 119, no. 8, pp. 1135-1145, 2009.

[25] K. E. Lewis, E. A. Kirk, T. O. McDonald et al., "Increase in serum amyloid A evoked by dietary cholesterol is associated with increased atherosclerosis in mice," Circulation, vol. 110, no. 5, pp. 540-545, 2004.

[26] J. A. van Diepen, J. F. P. Berbée, L. M. Havekes, and P. C. N. Rensen, "Interactions between inflammation and lipid metabolism: relevance for efficacy of anti-inflammatory drugs in the treatment of atherosclerosis," Atherosclerosis, vol. 228, no. 2, pp. 306-315, 2013.

[27] L. van Tits, R. Stienstra, P. van Lent, M. Netea, L. Joosten, and A. Stalenhoef, "Oxidized LDL enhances pro-inflammatory responses of alternatively activated M2 macrophages: a crucial role for Krüppel-like factor 2," Atherosclerosis, vol. 214, no. 2, pp. 345-349, 2011.

[28] A. Alipour, A. J. H. H. M. van Oostrom, A. Izraeljan et al., "Leukocyte activation by triglyceride-rich lipoproteins," Arteriosclerosis, Thrombosis, and Vascular Biology, vol. 28, no. 4, pp. 792-797, 2008.

[29] E. M. DeGoma, M. D. Davis, R. L. Dunbar, E. R. Mohler, P. Greenland, and B. French, "Discordance between non-HDLcholesterol and LDL-particle measurements: results from the Multi-Ethnic Study of Atherosclerosis," Atherosclerosis, vol. 229, no. 2, pp. 517-523, 2013.

[30] R. H. MacKey, P. Greenland, D. C. Goff Jr., D. Lloyd-Jones, C. T. Sibley, and S. Mora, "High-density lipoprotein cholesterol and particle concentrations, carotid atherosclerosis, and coronary events: MESA (multi-ethnic study of atherosclerosis)," Journal of the American College of Cardiology, vol. 60, no. 6, pp. 508-516, 2012.

[31] R. S. Rosenson, H. B. Brewer Jr., M. J. Chapman et al., "HDL measures, particle heterogeneity, proposed nomenclature, and relation to atherosclerotic cardiovascular events," Clinical Chemistry, vol. 57, no. 3, pp. 392-410, 2011.

[32] R. C. Hoogeveen, J. W. Gaubatz, W. Sun et al., "Small dense low-density lipoprotein-cholesterol concentrations predict risk for coronary heart disease: the Atherosclerosis Risk in Communities (ARIC) study," Arteriosclerosis, Thrombosis, and Vascular Biology, vol. 34, no. 5, pp. 1069-1077, 2014.

[33] T. Nishikura, S. Koba, Y. Yokota et al., "Elevated small dense low-density lipoprotein cholesterol as a predictor for future cardiovascular events in patients with stable coronary artery disease," Journal of Atherosclerosis and Thrombosis, vol. 21, no. 8, pp. 755-767, 2014.

[34] T. B. Grammer, M. E. Kleber, W. März et al., "Low-density lipoprotein particle diameter and mortality: the Ludwigshafen Risk and Cardiovascular Health Study," European Heart Journal, vol. 36, no. 1, pp. 31-38, 2015.

[35] J. T. Salonen, R. Salonen, K. Seppänen, R. Rauramaa, and J. Tuomilehto, "HDL, HDL2, and HDL3 subfractions, and the risk of acute myocardial infarction: a prospective population study in eastern finnish men," Circulation, vol. 84, no. 1, pp. 129-139, 1991.

[36] R. X. Xu, S. Li, X. L. Li et al., "High-density lipoprotein subfractions in relation with the severity of coronary artery disease: a Gensini score assessment," Journal of Clinical Lipidology, vol. 9, pp. 26-34, 2015. 


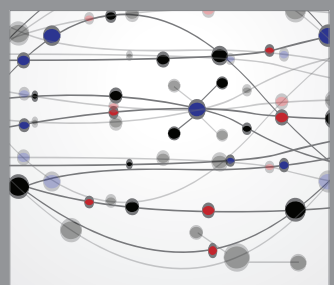

The Scientific World Journal
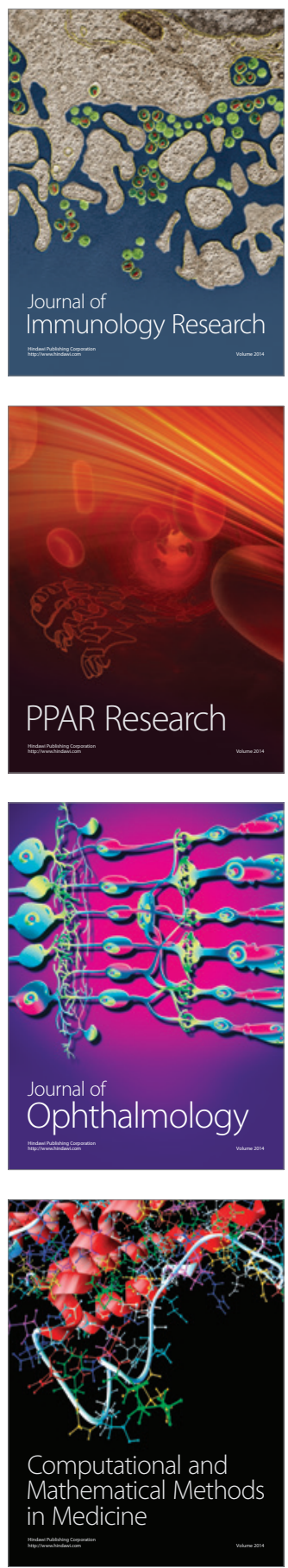

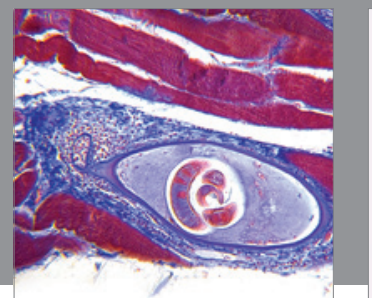

Gastroenterology

Research and Practice
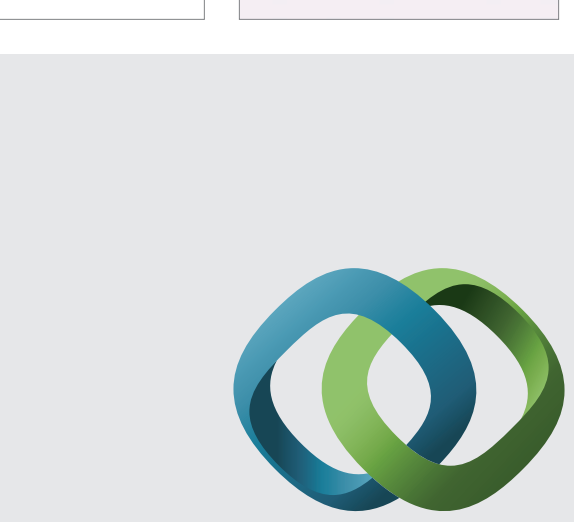

\section{Hindawi}

Submit your manuscripts at

http://www.hindawi.com
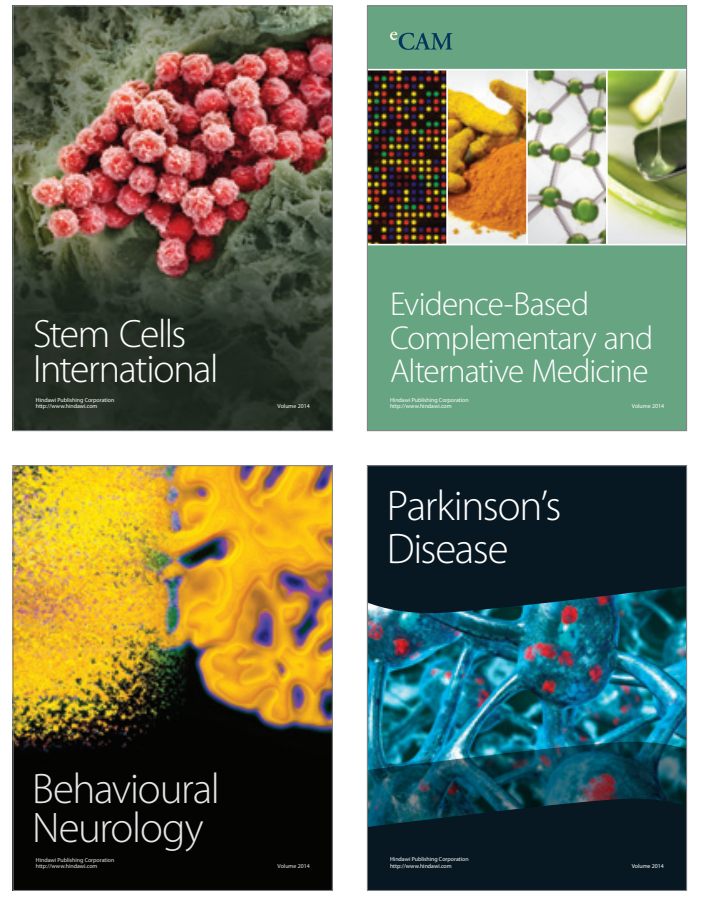
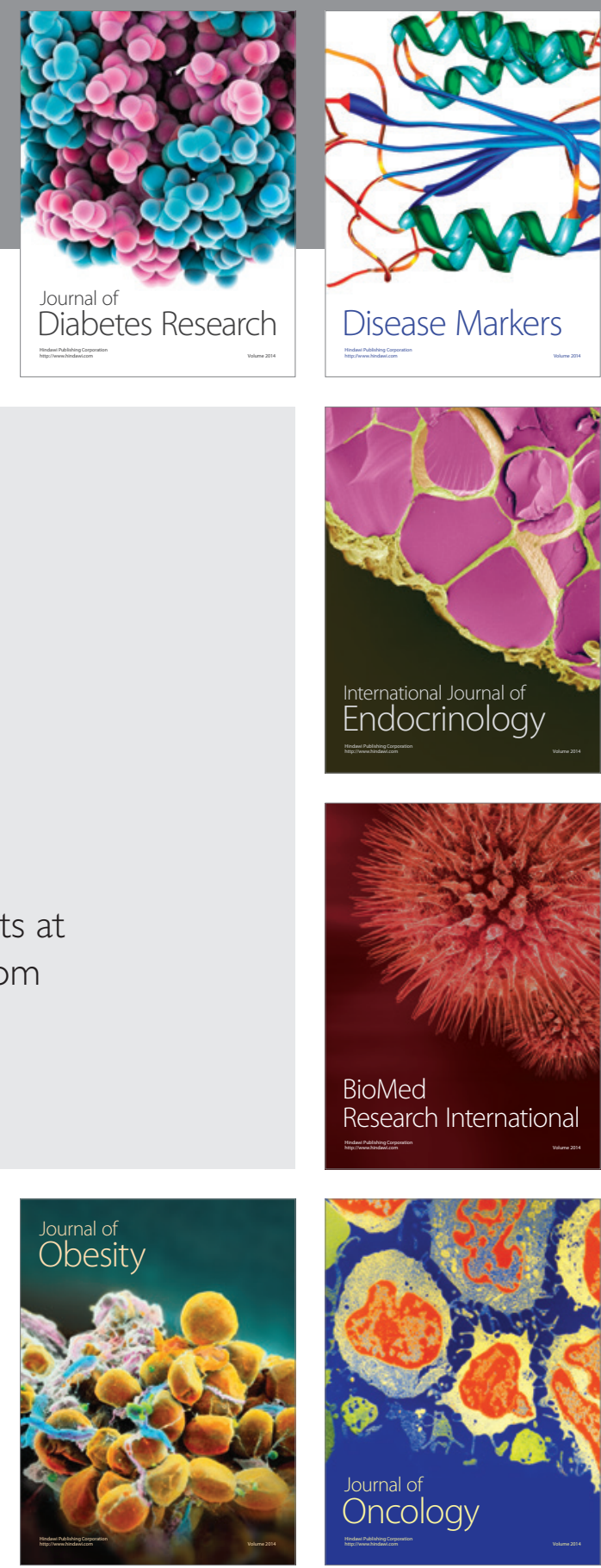

Disease Markers
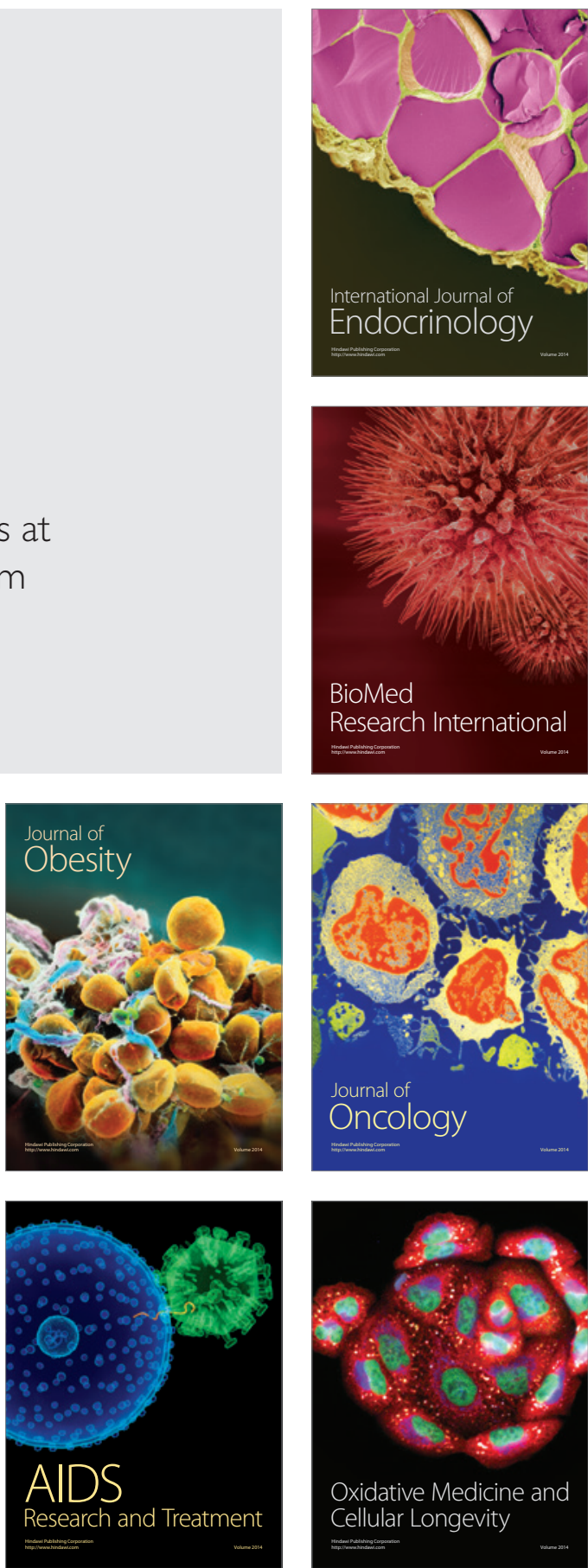